\title{
Mining and Environmental Monitoring at Open-Pit Mineral Deposits
}

\author{
Margarita Belova', Emiliia lakovleva ${ }^{2^{*}}$, Anton Popov ${ }^{1}$ \\ 1 Center of Geomechanicsand Issues of Mining Industry, Saint-Petersburg Mining University, 2, 21-st Line, \\ Vasilyevsky Island, 199106, St. Petersburg, Russian Federation \\ 2 Department of General Electrical Engineering, Saint-Petersburg Mining University, 2, 21-st Line, Vasilyevsky \\ Island, 199106, St. Petersburg, Russian Federation \\ * Corresponding author's e-mail: em88mi@gmail.com
}

\begin{abstract}
The paper deals with the arrangement of mining and environmental monitoring at open-pit mineral deposits using the electromagnetic geophysical method. The characteristic of the method of natural electromagnetic radiation (NEMR) discrete registration for searching geodynamically active faults to increase the safety of mining operations at ore deposits is given by the example of the apatite-nepheline deposit of the Khibiny rock massif. The existence of the dependence of the anomalous values of EMF in the Earth's pulse electromagnetic field on the stress-strain state of the massif on the basis of electrophysical phenomena occurring in the rock is substantiated. The efficiency of the method for search and mapping of geodynamic active faults is proven. In future, the use of this method will improve the effectiveness of measures taken to predict and prevent dangerous geodynamic phenomena.
\end{abstract}

Keywords: mining and environmental monitoring, natural electromagnetic radiation, pit, geophysical methods, disjunctive disturbances.

\section{INTRODUCTION}

In recent decades, the environment impact has increased multiple times. Due to the activities of mining enterprises, the depletion of natural resources, pollution and environmental intervention continue to grow (Kuranov 2012). Open-pit mining operations result in the destruction of biological components of landscape and cration of technogenic complexes. This leads to the disruption of significant areas of land, adjacent to pits and dumps, where the process of life development becomes difficult. As a result, pit excavations and dumps, in most cases, become lifeless territories after the completion of works. In addition, the disturbed areas, being centers of erosion processes, create environmental hazards.

Currently, there is an increase in the operating lifetime of pits and their depth, and the development of deposits with complex geological and geotechnical conditions is also carried out in a positive manner. Within this framework, the impact of instability of slopes can be catastrophic both from financial and environmental point of view. In particular, the emergency situations associated with the instability of edges, are very dangerous to people, equipment and adjacent areas. For example, the collapse of pit edges of the Korkinsky brown coal deposit (Figure 1), which took place in 2013, led to the resettlement of two nearby residential settlements, as the houses of local residents fell into the landslide zone (Zenkov et al. 2018). It is worth noting that this is not the first case of instability of the said pit edges. Deformations at the pitbegan in 1973.

In that situation the development of mining and underground construction, constantly requires the forecast of rock mass deformation (Kuranov et al. 2018); therefore, a timely forecast of any dangerous phenomena occurrence and monitoring the processes preceding them is of utmost importance. Thus, it is necessary to 


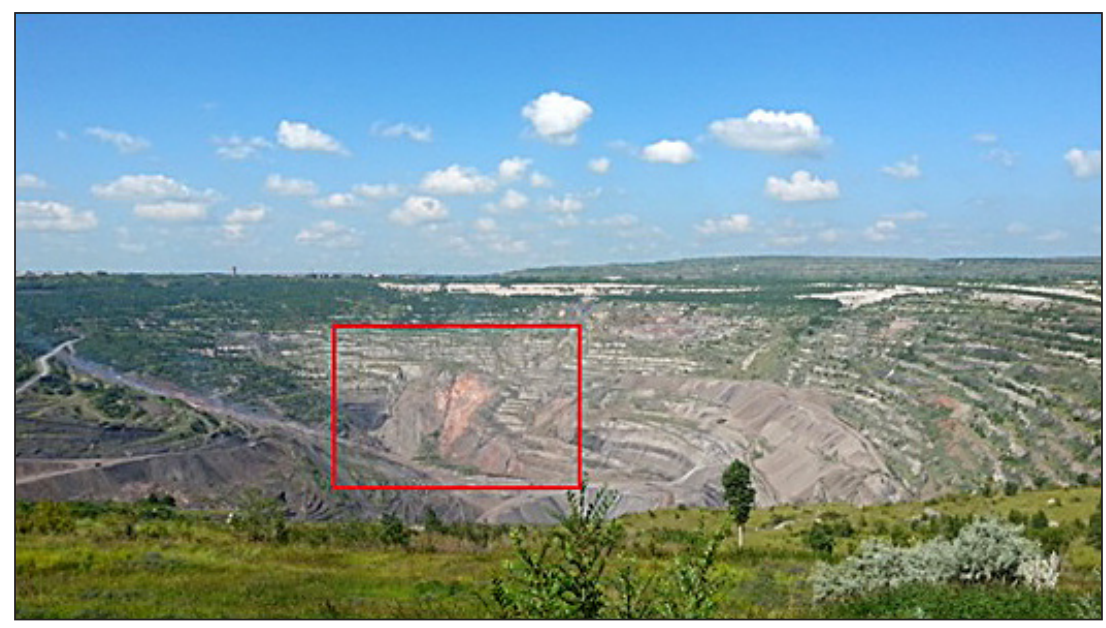

Figure 1. Rock fall at the Korkinsky brown coal deposit

simultaneously solve two inextricably connected tasks, namely, the rational use of all resources and preservation of natural amenities. In order to forecast the development of hazardous processes associated with mining operations, it is necessary to arrange mining and environmental monitoring studies in a timely manner. These studies include observations, assessment and forecast of mining operations negative influence on the environment as well as developing the recommendations to prevent this influence and the state of industrial safety during mining operations.

Among the tasks to be solved via conducting mining and environmental monitoring during mining operations, we shall specify the following: assessing and forecasting the state of environment, developing recommendations concerning prevention of man-made accidents, harmful influence of mining operations, rational use of mineral resources and subsoil protection. The tasks of mining and environmental monitoring are achieved via arranging a system of remote and terrestrial observations that ensure obtaining reliable qualitative information in a timely manner and to the extent required, analyzing this information and making appropriate decisions based on the results of the analysis.

The main task of mining and environmental monitoring is to detect hidden or weakly expressed disjunctive disturbances, presented in the form of intense fracturing zones, long-lived active disturbances that occur within the boundaries of blocks. This is due to the fact that dangerous geodynamic processes, capable of causing accidents, occur in these areas (Gurieva 2017). It is possible to detect the above-mentioned processes via recording natural electromagnetic radiation.
As is known, the geomagnetic field, among other abiotic factors, is one of the most important environmental factors, which is of utmost importance for vital activity of living organisms and plants.

This paper presents a method of detecting such zones and confirming their activity by studying natural electromagnetic radiation.

\section{MATERIAL AND METHODS}

During mining operations, dangerous engineering and geological processes, associated with the changes in the structure and parameters of the stress-strain state, occur. A special role is played by disjunctive disturbances. Currently, while performing geological and structural studies, the geological and geophysical features of disjunctive disturbances are subject to consideration to a lesser degree (Kharkevich 2006). The relief pattern of the Earth's surface serves as a criterion for detecting and mapping the major part of the faults. The areas of the Earth's surface, subject to the influence of modern tectonic processes, are characterized by a geodynamically unstable state of the earth's interior. This phenomenon is expressed by the movements of separate geological blocks that cause a local destruction of engineering facilities (including underground mines) as well as related seismic and seismoelectric phenomena (Hayakawa et al. 2002). The study of these phenomena is taken as a basis for geophysical methods of searching for zones of geodynamic activity of subsoil - there is a evidence of sharp anomalies in the structure of natural and induced physical fields near the centers of the stressed state of the massif and geodynamically active disturbances. 
Earlier, the Earth's electromagnetic noise was attributed to atmospheric thunderstorms, but the concept that electromagnetic noise is of atmospheric origin has been proven to be inconsistent (Malyshkov et al. 2015). Now, it is a well-known fact is that there are many processes in the lithosphere causing chaotic electromagnetic fields of different nature and intensity. Electromagnetic emission may start during crystal deformation prior to and during the nucleation phase of nanocracks (Greiling \& Obermeyer 2010). Natural alternating fields are regional alternating quasiharmonic and low frequency fields of cosmic (magnetotelluric) and atmospheric nature. Additionally, there are electric fields arising as the result of the electric activity of diffusion-adsorption, oxidation-reduction, filtration and electrode nature. Recording of the sum-total of these fields, revealing anomalous values and regularities will allow forecasting geodynamic phenomena.

However, the existing technologies and methods for mapping disjunctive disturbances are not described or proven indirectly by the nature of change in the natural electromagnetic field of the Earth. Therefore, one of the tasks of this paper is finding interconnection between the stability disturbance phenomenon and natural variations of the Earth's electromagnetic field.

As it is well-known, the sources of the natural electromagnetic field are the Earth, the Sun and other space objects. In addition to the natural field, there is an artificial electromagnetic field, which is created by various technical devices, such as power lines, transformer substations, electric and electronic equipment.

The natural electromagnetic field is characterized by changes in its activity in time (perturbations), having a selectable nature. There are three types of perturbations, in-phase, local and permanent. In-phase perturbations are characterized by the accidental occurrence and passing over the entire planet. Local perturbations are limited to a certain region on the Earth's surface. Permanent perturbations are observed continuously in some regions. The greatest changes in the field parameters are typical for in-phase and local perturbations. All the above-mentioned perturbations are caused by the changes in the magnetic activity of the Sun.

There are many processes in the lithosphere causing chaotic electromagnetic fields of different nature and intensity. Natural alternating fields are regional alternating quasi-harmonic and low frequency fields of cosmic (magnetotelluric) and atmospheric nature. Additionally, there are electric fields arising as the result of electric activity of diffusion-adsorption, oxidation-reduction, filtration and electrode nature.

Since a random superposition of these fields is fixed at every instant, resulting from the measurements of natural electromagnetic fields parameters, the task of determining the cause of the resultant field occurrence seems to be extremely difficult. In this paper, we present the assumptions of the most probable origin of the occurrence (Dolgiy \& Kataev 2015).

In rocks, inhomogeneities in the structure of soils are the sources of pulsed electromagnetic fields. As a result of the dynamoelectric phenomena, under the action of stress-related waves from the lower mantle, tidal forces, microseismic oscillations, wind and man-induced load, electromagnetic pulses, causing an electromagnetic background of lithospheric origin, arise on these sources (Dolgiy \& Kataev 2015). Such phenomena as the accumulation of electric charges in cracks during brittle failure or movement of rocks along the contacts of blocks, the subsequent relaxation of charges in the form of pulsed spark discharges, which create pulsed electromagnetic radiation in the rock mass, are known (Bezrodny et al. 2011). To this end, the change in the electromagnetic field parameters indicates the probability of occurrence of dangerous geodynamic processes in areas, where the field parameters differ from the background value.

The characteristics of electromagnetic field are as follows: frequency $f$, electric field strength $E$, magnetic field strength $H$, magnetic flux $\Phi$, power flow density $S$, and others. In this paper, we present the results of studying the derived value - the amplitude of the induced electromotive force in the inductance coil, placed in an alternating electromagnetic field.

In order to detect active disjunctive disturbances a method of measuring the amplitude of electromagnetic radiation (EMR) was used by applying the equipment that allows observing anomalies of the magnetic component of the AEMF (alternating electromagnetic field) using a magnetic-induction method. The advantages of the magnetic-induction method for recording AEMF anomalies, compared to other geophysical methods, applied to solve similar problems, above all include an optimal ratio between the rapidity and accuracy of measurements. 
The measurements were made with the APPA $109 \mathrm{~N}$ digital multimeter in the voltmeter mode, which specifies the electromotive force induced by the field in the sensor (antenna). During the studies, the "IA-Wave" antenna, recording the magnetic component of the electromagnetic field with a maximum signal perception spectrum of $32 \mathrm{kHz}$, was used as the signal receiver.

The methodology depth in the selected frequency range lies within the first tens of meters, and in the media with high specific electric resistanceit is slightly more than a hundred meters, which is enough to accomplish the tasks of detecting the centers of stressed state of walls of underground mines. When electromagnetic waves pass through the rocks from a deep source, the highfrequency component of the natural alternating electromagnetic field decays relatively rapidly with distance, and the low-frequency component can be better recorded at the receiving point of the alternating electromagnetic field on the surface. The proportion of the observed signal is the result of the induction of secondary electric currents in the conductive parts of the rocks, which can also be considered as a criterion for detecting the weakened zones of underground mines (Popov et al. 2017).

This central frequency of the antenna allows one to reduce the influence on the measuring results, caused by atmospherics (frequency range is $1-20 \mathrm{kHz}$ ), distant radio stations (frequency range is from hundreds of kilohertz and above) and other disturbances of anthropogenic origin, but to eliminate their influence completely is impossible.

Due to the fact that during the study the value of electromotive force was measured, the magnetic component of the electromagnetic field is of main interest. The natural electromagnetic field of the Earth was taken as a background value. The determination of background values was carried out on the basis of statistical processing of the data obtained from the profile.

According to the electromagnetic induction law, with any change in the magnetic flux through the conducting loop, an electric current is produced in this loop:

$$
E=-n \frac{d \Phi}{d t}
$$

where: $n$ is the number of turns in the coil, and the magnetic flux through the surface is determined as follows:

$$
\Phi=B S \cos \alpha
$$

where: $B$ is the magnetic induction vector, $S$ is the area of the closed loop, and $\alpha$ is an angle between the direction of the magnetic induction vector and the normal to the loop area.

The magnetic flux value, and hence, the induced electromotive force value depends on the angle $\alpha$; therefore, due to the unknown direction of the magnetic lines, the measurements were carried out in three directions $-\mathrm{X}, \mathrm{Y}, \mathrm{Z}$. On the basis of the measuring results, it is possible to assume the direction of the magnetic field lines. The smallest value of electromotive forcewill be reached by the parallel arrangement of the loop and the magnetic field lines, when the angle $\alpha$ is equal to $90^{\circ}$.

\section{RESULTS}

The deposits under consideration are located in the southern end of the Khibiny massif. The enclosing rocks of the deposits are characterized by a high index of strength and elasticity. The ultimate compression strength of rocks is $120-250$ $\mathrm{MPa}$, while the tensile strength is $5-20 \mathrm{MPa}$. Accordingly, mine formations also have high indices of brittleness coefficient. In addition, taking into account the block structure of the massif and having analyzed the literature sources as well as the results of field studies within the deposits, the rock mass of the deposits is characterized by the development of 4 to 5 natural fracture systems, with drilling-induced fractures accounted for. The large-block cracks, the length of which extends over hundreds and tens of meters, are the most dangerous; the value of their opening varies within the range of $1-50 \mathrm{~mm}$, wherein the cracks are filled with hydrothermal minerals. The operation of deposits is also complicated by the development of hydrothermal processes, varying in scale, i.e. sprocketization. Such zones of weakness, according to the deposit operational control records, can be detected at depths of up to 900 meters, representing steeply dipping zones of secondary changes, with length up to hundreds of meters (Popov et al. 2017).

Strong brittle rocks and wide distribution of zones of weakened rocks enable to classify deposits as dangerous by mining-tectonic impacts. 
Referring to the above-mentioned information, the research work, aimed to detect weakened zones, in terms of sustainability, is extremely relevant.

While surveying the pit edges, the methodology used included discrete measurements of the amplitude of the magnetic component of the alternating electromagnetic field on the Earth's surface, with an exposure time of 10s along profiles, parallel to the pit edges. At each point of the profile, 3 measurements were made with different orientations of the magnetic moment of the antenna: downwards, frontwards along the profile and across the profile of observations. The interval between study points for each profile was selected individually, based on the expected crosssectional dimensions of the anomalies.

While mapping disjunctive disturbances, revealed according to geological data, the vertical component - Az (the antenna is directed downwards) is the most informative. According to the anomalies of $\mathrm{Az}$ (expressed in millivolts via the proportional value of the electromotive force induced in the antenna), most of the tectonic disturbances that can be seen in the pit edges are fixed. Having analyzed the whole set of measurements, the background range of the values was determined as not exceeding 70, 80 and $90 \mathrm{mV}$ for the three profiles, correspondingly, and an anomalous increase in the signal amplitude by $\sim 20 \%$ is assumed to be significantly anomalous compared to the background values (Table 1). The difference in the average values for different profiles, the works on which are performed at different times, can be referred to the presence of diurnal variations of the electromagnetic radiation (Basov et al. 2015).
Profile 3, extended along three potentially dangerous areas was taken for the purpose of illustration in this work. The total length of the profile is 60 meters; the measurements were made at a pitch of 1 meter (Fig. 2).

This profile includes three areas with strongly expressed structure inhomogeneity in the form of fault tectonics, dykes of lamprophyre composition, and zones of intense fracturing with intense water ingress. According to the authors' assumption, all three areas should have given high-contrast anomalies of the parameters studied while measuring, which was shown by the works performed (Fig. 3).

The diagram in Figure 3 shows that the detected anomalies of electromotive force $\mathrm{Az}$ (when the antenna is oriented "downwards") correlate well with the projections to the profile line of two branches of tectonic disturbance. The dyke, whilst it is characterized only by small-amplitude anomalies, related mainly to its contacts with enclosing rocks, is fixed nevertheless. The most visually striking on the profile are anomalies spatially associated with flooded fractured zones, which is due to the complex influence of reduced resistance of watered rocks (respectively, due to the induction of electric currents therein and the formation of stronger secondary fields), as well as to a larger value of own filtration potentials and the formation of a heterogeneous double electric layer on the boundary of rock particles and pore solutions. Measurements for this profile were made after precipitation, which was a favorable factor for specifying fracture zones, over which the water inflow discharge takes place.

Table 1. Basic statistics for some profiles of magnetic induction survey on the bench areas of the pit "Centralny"

\begin{tabular}{|c|c|c|}
\hline \multirow{2}{*}{ Profile, No. } & Parameters & $\begin{array}{c}\text { Electromotive force induced by the vertical } \\
\text { component Az of the magnetic component of } \\
\text { the alternating electromagnetic field, } \mathrm{mV}\end{array}$ \\
\hline \multirow{3}{*}{ Profile 1 } & background values & up to 80 \\
\cline { 2 - 3 } & average value & 70.61 \\
\cline { 2 - 3 } & amplitude of local anomalies & up to 15 \\
\hline \multirow{3}{*}{ Profile 2 } & background values & up to 70 \\
\cline { 2 - 3 } & average value & 71.72 \\
\hline \multirow{2}{*}{ Profile 3 } & amplitude of local anomalies & $5-15$ \\
\cline { 2 - 3 } & background values & up to 90 \\
\cline { 2 - 3 } & average value & 78.93 \\
\cline { 2 - 3 } & amplitude of local anomalies & $10-50$ \\
\hline
\end{tabular}




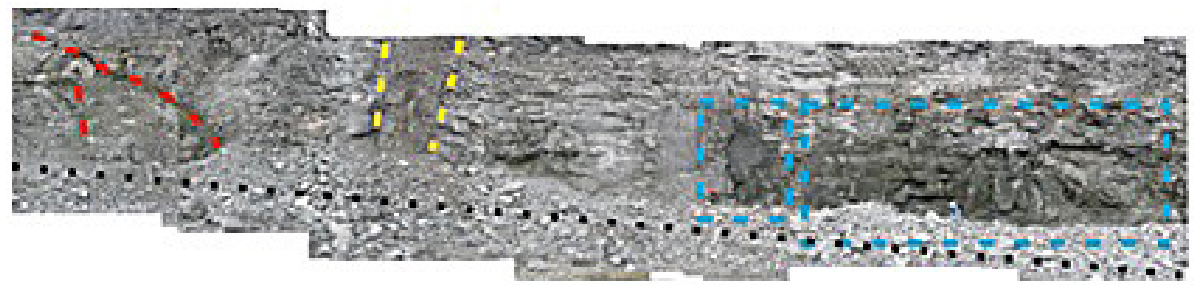

Figure 2. Photo collage of the pit edge, along which the measurements were taken on the support profile. The measurement points are shown with black dots. The scale is slightly distorted due to the peculiarities of photography.

Red line - tectonic disturbance; Blue line - boundaries of the fracture zone controlled by the dyke; Yellow line the area with visible water inflow from the cracks in the pit edge.

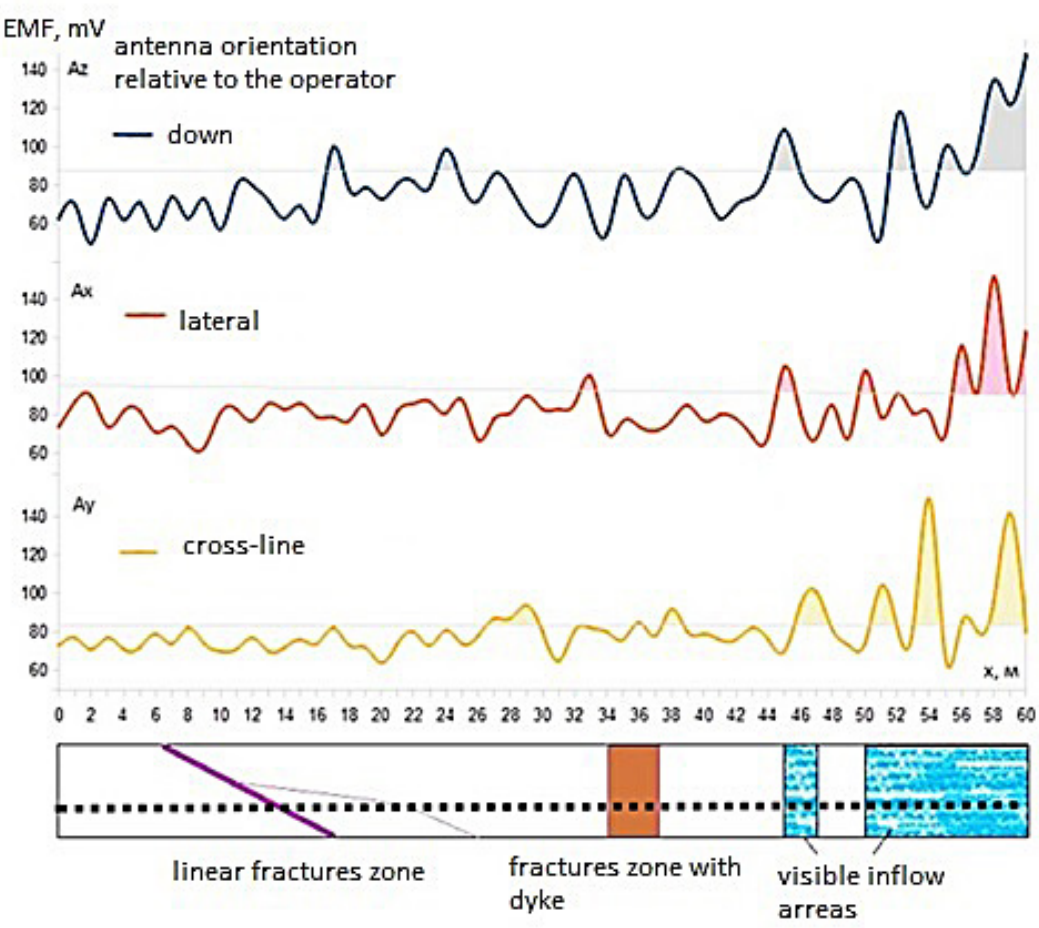

Figure 3. The results of observations along the profile 3 and the diagram of tectonic disturbances of the nearest edge of the pit

\section{DISCUSSION}

Three directions of the antenna orientation were used for measurements due to the fact that it is not possible to determine the direction of propagation of magnetic field lines. With this method, it is possible to achieve the necessary variability of the measurement results of the EMF induced in the antenna, since the measurements are made at three different angles $\boldsymbol{\alpha}$. The results of the conducted measurements showed that the angle $\boldsymbol{\alpha}$ reaches the lowest value, and the EMF reaches the highest value when the antenna is directed downwards.
The anomalies caused by the presence of watered fractured zones are manifested most clearly. This is due to the complex effect of reduced resistance of watered rocks (respectively, easier induction of electric currents in them), the presence of its own filtration potentials and the formation of heterogeneous DEL (double electric layer).

The complexity of the dike mapping is explained by the fact that in this case the fault is filled with monchikite; therefore, according to the magnetic component of the alternating electromagnetic field data it is only possible to register the boundaries of the body; however, for this purpose the profiling step must be rather small. 


\section{CONCLUSION}

The considered results of mapping potentially geodynamically active zones via measurement of the alternating electromagnetic field parameters by the induction method are in favor of the high efficiency of the method of discrete registration of electromagnetic field components for detecting and mapping disjunctive disturbances both in the pit edges and in underground mines. The average repeatability of the measuring results for the alternating electromagnetic field parameters and data of geological investigations made about $85 \%$.

Having analyzed the results of the works performed, it is possible to predict similar efficiency of use of the considered methodology and instrumental base for mapping zones of geodynamically active faults at other ore deposits and facilities with a significantly expressed fault-block structure. The authors see the possibility of improving the methodology by conducting measurements jointly at two frequencies (which can be implemented via two different antennas); in the future it will eliminate the influence on the survey results, caused by a number of negative factors and increase the reliability of the results.

Thus, the challenging issue of detecting potentially dangerous zones of underground mines at a timely manner can be solved via studying the amplitude of natural electromagnetic radiation. The proposed methodology can become a part of a complex system of environmental monitoring for assessing and forecasting the state of the natural environment in open pit mining areas. In the future this will allow reducing negative influence of mining activities on the environment, reducing the possibility of accidents at the facilities that cause damage to the equipment, people and adjacent areas, since the most important point of mining and environmental monitoring is forecasting the consequences of exploitation of natural resources.

\section{REFERENCES}

1. Basov A.D., Romanevich K.V., Shlyaev S.A. 2015. Monitoring system of continuous recording of natural electromagnetic radiation in operating transport tunnels. Materials of the international scientific and practical conference and exhibition EAGO "Engineering, coal and ore geophysics.Current state and development prospects", 76-80.
2. Bezrodny K.P., Basov A.D., Romanevich K.V. 2011. Control of the stress-strain state of the rock mass during tunnel construction using natural electromagnetic radiation method.Izvestiya of Tula State University.Earth sciences, Issue 1, 227-234.

3. Dolgiy M.E., Kataev S.G. 2015. Studying the natural pulsed electromagnetic field of the Earth. Bulletin of Tomsk State University, No. 2 (34), 61-66.

4. Gurieva A.A. 2017.Sustainable development of oreraw materials and enhanced capacities of apatitjsc based on best engineering solutions. Mining University collection of scientific papers, V 228, 662 -673.

5. Hayakawa M., Molchanov O.A., Nickolaenko A.P. 2002. Model variations in atmospheric radio noise caused by pre-seismic modification of tropospheric conductivity profile.seismo electromagnetics. Lithosphere Atmosphere-Ionosphere Coupling, 349-352.

6. Kharkevich, A.S. 2006. Forecasting of the position of zones of geodynamic activity of coal seams by geophysical methods in the mines of Kuzbass. Collection of scientific papers of All-Russian Research Institute of Mining Geomechanics and Survey, 290-299.

7. Kuranov A.D. 2012. Determination of the parameters of the spatial strength condition of the mounting sites of ore careers in high-spinded scalar massives. Mining University collection of scientific papers.V 199, $111-117$.

8. Kuranov A.D., Zuev B.Y., Istomin R.S. 2018. The forecast deformations of the ground surface during mining under protected objects. Innovation-Based Development of the Mineral Resources Sector: Challenges and Prospects - 11th conference of the Russian-German Raw Materials, 39-50

9. Malyshkov Y.P., Malyshkov S.Y., Gordeev V.F., Shtalin S.G., Polivach V.I., Krutikov V.A., Zaderigolova M.M. 2015. Horizons in World Physics, Volume 283. Nova Science Publishers Inc., New York.

10. Popov A.L., Parchimchik M.V., Senchina N.P., Idiyatullin M.M. 2017. Localization of potentially dangerous zones of rock massif by the results of studying natural electromagnetic radiation. VIII International Scientific and Practical Conference "Innovative approaches in mining enterprises design: geomechanical design support of mining operations", 238-240.

11. Reinhard O. Greiling, Hennes Obermeyer. 2010. Natural Electromagnetic Radiation (EMR) and its Application in Structural Geology and Neotectonics. Journal of the Geological Society of India, vol. 75, 278-288.

12. Zenkov I.V., Nefedov B.N., Kiriushina E.V., Zayatz V.V. 2018. Results of disturbed lands environmental condition remote monitoring in "Korkinsky” open-pit mine. Ugol', Issue 9,99-101. 